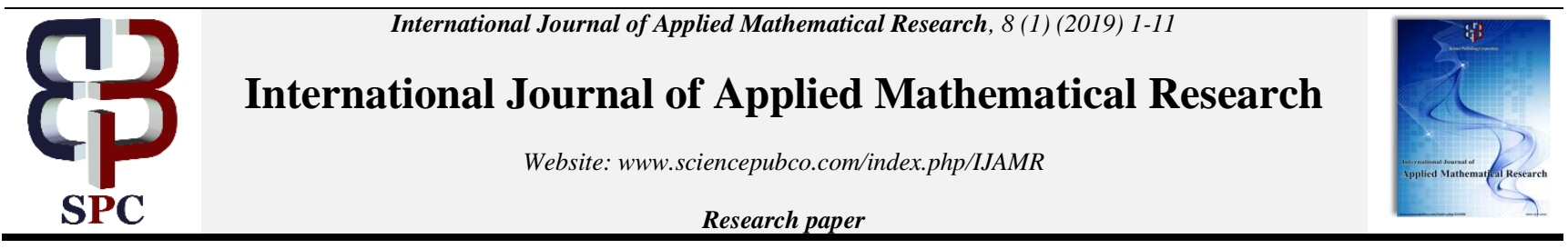

\title{
Modeling HIV/AIDS co infection with malaria and tuberculosis: the role of treatment and counseling
}

\author{
Mark O. Okongo * \\ Department of Physical sciences, Chuka University Kenya \\ *Corresponding author E-mail: marikookongo@gmail.com
}

\begin{abstract}
HIV/AIDS remains one of the leading causes of death in the world with its effects most devastating in Sub Saharan Africa due to its dual infection with opportunistic infections especially malaria and tuberculosis. This study presents a co infection deterministic model defined by a system of ordinary deferential equations for HIV/AIDS, malaria and tuberculosis. The HIV/AIDS only model is analyzed to determine the conditions for the stability of the equilibria points and assess the role of treatment and counseling in con-trolling the spread of the infections. This study shows that effective counseling reduces the value of the reproduction number for HIV/AIDS ( $\left.\mathrm{R}_{\mathrm{H}}\right)$ to less than unity eliminating the HIV/AIDS problem. Numerical simulations show that applying anti-retroviral treatment (ARV'S) without effective counseling increases the value of $\mathrm{R}_{\mathrm{H}}$, worsening the HIV/AIDS problem, however ARV treatment coupled with effective counseling reduces the value of $R_{H}$ to a level below one eliminating the disease. The study further shows that when the proportion of those receiving ARV treatment without effective counseling increases, the value of $R_{H}$ also increases to a level above one. However effective counseling maintains the value of $\mathrm{R}_{\mathrm{H}}$ below unity therefore strategies for the control of HIV/AIDS should emphasize counseling and not only treatment.
\end{abstract}

Keywords: HIV/AIDS - TB and Malaria; Equilibria; Stability; Bifurcation; Sensitivity, Counseling, Treatment.

\section{Introduction}

Research at the interface of mathematics and biology is increasing, and virtually any advance in disease dynamics today requires a sophisticated mathematical approach in order to map out the parameters necessary for control and containment of epidemic outbreaks. Infectious diseases, alongside cardiovascular diseases and cancer have been the main threat to human health. Acute and chronic respiratory diseases, especially pulmonary tuberculosis, malaria and HIV/AIDS are responsible for a large portion of mortality especially in developing countries [16].

Globally HIV/AIDS has killed more than 35 million people since it was first discovered in 1981 and almost 70 million people have been infected with the HIV/AIDS virus making it one of the most destructive epidemics in recorded history [22]. It remains one of the leading causes of death in the world with its effects most devastating in sub Saharan Africa. One of the key factors that fuels the high incidence of HIV/AIDS in Sub Saharan Africa is its dual infection with malaria and tuberculosis [16].

World Health Organization statistics show that tuberculosis (TB) is the most common illness and the leading cause of death among people living with HIV/AIDS, accounting for one in four HIV/AIDS related deaths and at least one-third of the 34 million people living with HIV/AIDS worldwide are infected with latent TB. Persons co infected with TB and HIV/AIDS are 21-34 times more likely to develop active TB disease than persons without HIV/AIDS. In 2011, there were an estimated 1.1 million HIV/AIDS positive new TB cases globally and about 79 percent of these people live in Sub-Saharan Africa [22]. According to the World Health Organization report of April 2008, malaria increases the viral load in HIV/AIDS patients. Conversely HIV/AIDS increases the risk of malaria infection and accelerate the development of clinical symptoms of malaria with the greatest impact on the immune suppressed persons [22]. Ever since the co infections were recorded, malaria has seen a 28 percent increase in its prevalence and malaria related death rates have also nearly doubled for those with co infections [7]. The co infection between malaria and HIV-1 is the commonest in Sub-Saharan Africa and, to a lesser extent, in other developing countries. It is estimated that 22 million Africans are infected with HIV-1, and around 500 million are suffering from malaria annually [22].

Hohman and Kami [10], discovered that HIV/AIDS and malaria have similar global distributions. The discovery motivated a study on the impact of HIV/AIDS and malaria co infection and established that globally, 500 million people are infected with malaria annually resulting in one million deaths yearly. Thirty-three million people get infected with HIV/AIDS and 2 million die from it every year. The study further showed that those with HIV/AIDS have more frequent episodes of symptomatic malaria and that malaria increases HIV/AIDS plasma viral load and decrease CD4 ${ }^{+}$cells. During episodes of parasitemia, HIV/AIDS infected people have an increase in viremia leading to potential increase in risk of HIV/AIDS transmission. A comparison of the geographical distributions of HIV/AIDS, $\mathrm{TB}$ and malaria especially in Africa, reveal that these three diseases have similar geographical distributions suggesting a possible existence of HIV/AIDS, TB and malaria co infection. This may be due to shared risk factors and/or the presence of opportunistic infections. 
Audu et al. [4] investigated the possible impact of co infections of tuberculosis and malaria on the CD4+ cell counts of HIV/AIDS patients and established the following: The healthy control group recorded a median CD4 ${ }^{+}$cell counts of 789 cells/ul $\left(789\right.$ cells per mm $^{3}$ of blood); subjects infected with HIV/AIDS only recorded a median CD4 ${ }^{+}$cell counts of 386 cell/ul; subjects co infected with HIV/AIDS and $\mathrm{TB}$ recorded a median $\mathrm{CD} 4^{+}$cell counts of 268 cell/ul; subjects co infected with HIV/AIDS and malaria recorded a median CD4 ${ }^{+}$ cell counts of 211 cell/ul and those co infected with HIV/AIDS, malaria and TB recorded the lowest median CD4 $4^{+}$cell counts of 182 cell/ul.

Motivated by these findings, this study aims at developing a deterministic model exploring the joint dynamics of the simultaneous co infections of HIV/AIDS, TB and malaria incorporating treatment and counseling for the HIV/AIDS infected population. It represents the first deterministic mathematical model incorporating HIV/AIDS, TB and Malaria co infections within a single model to gain insights into their combined transmission dynamics and determine effective control strategies.

\section{Model formulation and description}

To study the dynamics of HIV/AIDS, malaria and TB co infection, a deterministic model is formulated described by a system of ordinary differential equations. The model sub-divide the human population into the following epidemiological classes: $S_{H}(t)-S_{u s c e p t i b l e}$ population at time $t, \mathrm{I}_{\mathrm{M}}(\mathrm{t})$ - Malaria infectives at time $\mathrm{t}, \mathrm{I}_{\mathrm{H}}(\mathrm{t})$ - HIV cases at time $\mathrm{t}, \mathrm{I}_{\mathrm{A}}(\mathrm{t})$ - AIDS cases at time $t$, $\mathrm{IT}_{\mathrm{T}}(\mathrm{t})$ - TB cases at time $t$. $\operatorname{Ihm}(t)$ - Those co infected with malaria and HIV at time $t$, IAM $(t)$ - Those co infected with malaria and AIDS at time $t$, IMT (t) - Those co infected with malaria and TB at time $t, \mathrm{I}_{\mathrm{HT}}(\mathrm{t})$ - Those co infected with HIV and TB at time $\mathrm{t}, \mathrm{I}_{\mathrm{AT}}(\mathrm{t})$ - Those co infected with AIDS and TB at time $t$, I $\mathrm{I}_{\text {Hмт }}(\mathrm{t})$ - Those co infected with HIV, Malaria and TB at time $t$, $\mathrm{I}_{\mathrm{AMT}}(\mathrm{t})$ - Those co infected with AIDS, Malaria and TB at time $t$. The total human population $\left(\mathrm{N}_{H}(\mathrm{t})\right)$ is therefore denoted by:

$\mathrm{N}_{H}(\mathrm{t})=\mathrm{S}_{\mathrm{H}}(\mathrm{t})+\mathrm{IM}_{\mathrm{M}}(\mathrm{t})+\mathrm{I}_{\mathrm{H}}(\mathrm{t})+\mathrm{I}_{\mathrm{A}}(\mathrm{t})+\mathrm{IT}_{\mathrm{T}}(\mathrm{t})+\mathrm{IHM}_{\mathrm{H}}(\mathrm{t})+\mathrm{I}_{\mathrm{AM}}(\mathrm{t})+\mathrm{I}_{\mathrm{MT}}(\mathrm{t})+\mathrm{I}_{\mathrm{HT}}(\mathrm{t})+\mathrm{I}_{\mathrm{AT}}(\mathrm{t})+\mathrm{IHMT}_{\mathrm{T}}(\mathrm{t})+\mathrm{I}_{\text {AMT }}(\mathrm{t})$. The vector (mosquito) population at time $\mathrm{t}$ denoted by $N_{v}(t)$ is sub-divided into the following classes: $S_{v}(t)$ - Vector susceptibles at time $t$, Iv (t) - Vector infectives at time $t$. The total vector population $\mathrm{NV}_{v}(\mathrm{t})$ is given by $\mathrm{N}_{\mathrm{v}}(\mathrm{t})=\mathrm{S}_{\mathrm{v}}(\mathrm{t})+\mathrm{Iv}(\mathrm{t})$.

\subsection{Definition of parameters}

It is assumed that susceptible humans are recruited into the population at a constant rate either by birth or recovery from malaria and TB. They acquire infection with either HIV/AIDS, malaria or TB and move to the infectious classes. Susceptible mosquitoes are recruited into the mosquito population at a constant rate. They acquire malaria infection following a blood meal feeding on infected malaria humans, becomes infectious and move to the infectious class. The recruitment rate of humans into the susceptible population is denoted by $\Lambda_{\mathrm{H}}$ while that of vectors (mosquitoes) is denoted by $\Lambda_{\mathrm{v}}$ and are both assumed to be constant. The natural death rate of humans is given by $d_{n}$ while that of vectors is given by $d_{v}$. The death rates due to AIDS, malaria and TB in humans are $d_{a}, d_{m}$ and $d_{t}$ respectively. The parameters $d_{a m}, d_{m t}, d_{a t}$ and $d_{a m t}$ account for the combined death rates in the $I_{A M}, I_{M} T$, $I_{A T}$ and $I_{A M T}$ classes respectively. The parameters $r_{m}$ and $r_{t}$ are the recovery rates from malaria and TB respectively due to effective treatment. It is assumed that the recovered individuals do not acquire temporary immunity to either or both diseases thus become susceptible again. The model assumes that susceptible humans cannot simultaneously get infected with malaria, HIV/AIDS and TB since the transmission mechanics are completely different for the three diseases. The model further assumes that humans acquire HIV/AIDS through sexual contacts between an infective and a susceptible. The average force of infection for HIV/AIDS denoted $\lambda_{\text {ah }}$ is given by.

$\lambda_{a h}=\frac{\beta_{a}(1-\delta) c_{1}\left(I_{H}+I_{H M}+I_{H T}\right)}{N_{H}}$

where $\beta_{\mathrm{a}}$ is the average transmission probability of HIV/AIDS between an infective and a susceptible per sexual contact and $c_{1}$ is the per capita number of sexual contacts of susceptible humans with HIV/AIDS infected individuals per unit time. The parameter $\delta$ measures the effectiveness of counseling through condom use and a reduction in the number of sexual partners, where $0 \leq \delta \leq 1$. Effective counseling reduces the value of the parameter $\mathrm{c}_{1}$. The model assumes that the classes $\mathrm{I}_{\mathrm{HMT}}, \mathrm{I}_{\mathrm{A}}, \mathrm{I}_{\mathrm{AM}}, \mathrm{I}_{\mathrm{AT}}$ and $\mathrm{I}_{\mathrm{AMT}}$ do not transmit the virus due to acute ill health and noticeable AIDS symptoms. Define $\alpha_{1}$ as the number of bites per human per mosquito (biting rate of mosquitoes), $\beta_{\mathrm{m}}$ as the transmission probability of malaria in humans per bite thus the force of infection with malaria for humans, denoted $\lambda_{\text {mh }}$ is given by.

$\lambda_{m h}=\frac{\alpha_{1} \beta_{m} I_{V}}{N_{H}}$

Whereas the average force of infection with malaria for vectors, denoted $\lambda_{\mathrm{mv}}$ is given by

$\lambda_{m v}=\frac{\alpha_{1} \beta_{v}\left(I_{M}+I_{H M}+I_{M T}+I_{A M}+I_{H M T}+I_{A M T}\right)}{N_{H}}$

Where $\beta_{\mathrm{v}}$ is the transmission probability of malaria in vectors from any infected human Finally the average force of infection for TB denoted $\lambda_{\text {th }}$ is given by

$\lambda_{t h}=\frac{\beta_{t} c_{2}\left(I_{T}+I_{H T}+I_{M T}+I_{H M T}+I_{A M T}+I_{A T}\right)}{N_{H}}$

where $\beta_{\mathrm{t}}$ is the transmission probability of TB in humans and $\mathrm{c}_{2}$ is the average per capita contact rate of susceptible humans with TB infected individuals.

The rate of progression from HIV to AIDS for the untreated HIV cases is $p$. The parameters $\theta_{1} p, \theta_{2} p$ and $\theta_{3} p$ account for increased rates of progression to AIDS for individuals co infected with HIV - TB, HIV - malaria and HIV - malaria - TB respectively where $\theta_{1}<\theta_{2}<\theta_{3}$. Define $\alpha$ as the proportion of the HIV/AIDS infectives receiving effective treatment. This involves the administration of ARV'S that keeps the HIV patients from progressing to AIDS while transferring the AIDS patients back to the HIV classes. The modification 
parameters $e_{m}^{h}, e_{t}^{h}$ and $e_{m t}^{h}$ account for the reduced susceptibility to infection with HIV for individuals in the IM , IT and the IMT classes respectively due to reduced sexual activity as a result of ill health where $e_{h}^{m}<1, e_{t}^{h}<1, e_{m t}^{h} \ll 1$. The parameters $e_{a}^{m}, e_{h}^{m}, e_{h t}^{m}, e_{a t}^{m}$, account for the increased susceptibility to infection with malaria for individuals already infected with AIDS, HIV, HIV - TB and AIDS TB respectively due to suppressed immune system where $e_{a}^{m}>1, e_{h}^{m}>1, e_{h t}^{m}>1, e_{a t}^{m}>1$. It is also clear that $e_{a}^{m}<e_{a t}^{m}$ and $e_{h}^{m}<e_{h t}^{m}$. The parameters $e_{h}^{t}, e_{a}^{t}, e_{m h}^{t}$, and $e_{m h}^{t}$ account for the increased susceptibility to infection with TB for individuals already infected with HIV, AIDS, HIV - malaria and AIDS - malaria respectively due to suppressed immune system where $e_{h}^{t}>1, e_{a}^{t}>1, e_{m h}^{t}>1, e_{a m}^{t}>1$. Again $e_{h}^{t}>e_{m h}^{t}$ and $e_{a}^{t}<e_{a m}^{t}$. Malaria and TB does not lead to the depletion of the CD4 ${ }^{+}$cell counts, However their association with HIV/AIDS leads to a significant reduction in the $\mathrm{CD}^{+}$cell counts within an individual leading to faster progression to AIDS.

\subsection{The model equations}

Combining all the aforementioned assumptions and definitions, the model for the transmission dynamics of HIV/AIDS, TB and malaria is given by the following system of differential equations:

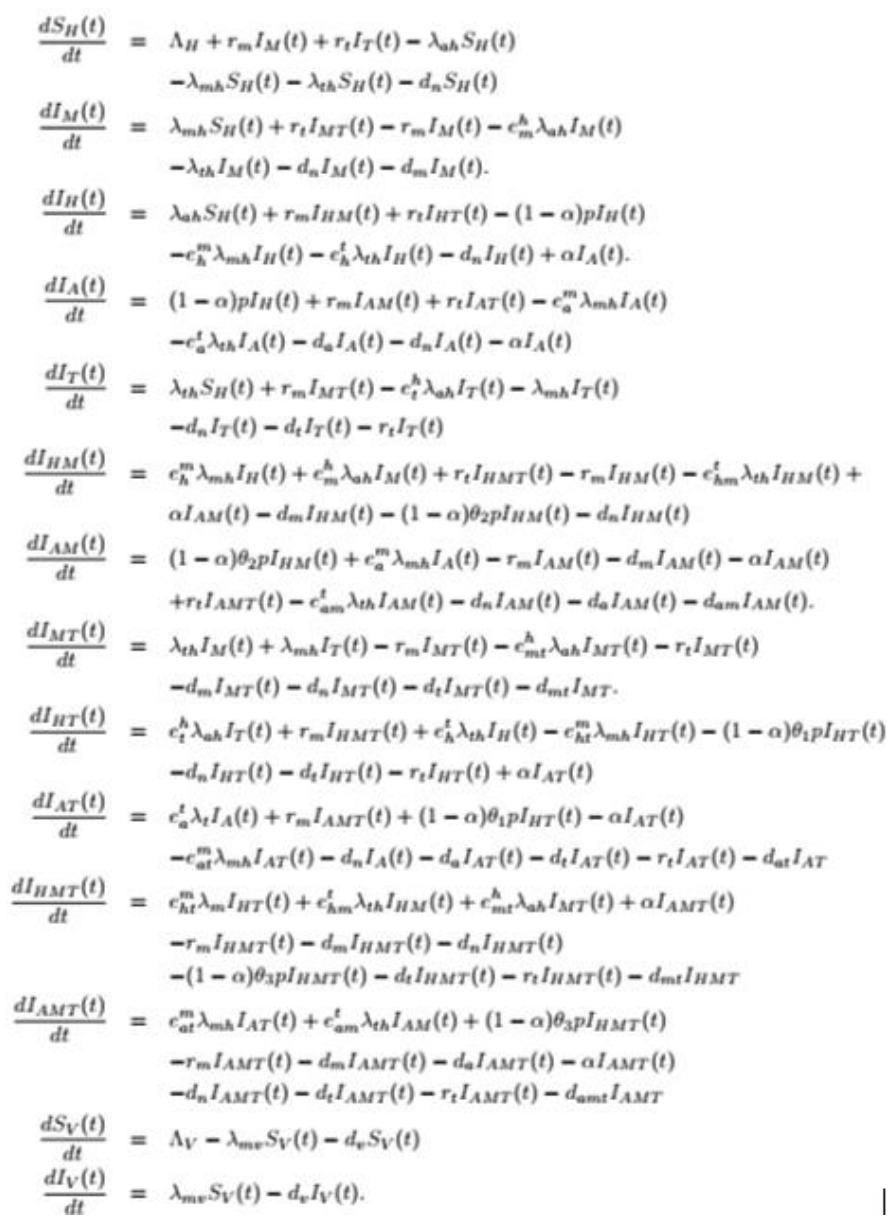

\section{Positivity and boundedness of solutions}

The model system 2.2.1 describes living populations therefore the associated state variables are non-negative for all time $t \geq 0$. The solutions of this model with positive initial data therefore remain positive for all time $t \geq 0$.

Lemma 3.1. Let the initial data set be $\left\{\mathrm{S}_{\mathrm{H}}(0) ; \mathrm{S}_{\mathrm{V}}(0)>0\right) ;\left(\mathrm{I}_{\mathrm{M}}(0) ; \mathrm{I}_{\mathrm{H}}(0) ; \mathrm{I}_{\mathrm{A}}(0) ; \mathrm{I}_{\mathrm{T}}(0) ; \mathrm{I}_{\mathrm{HM}}(0) ; \mathrm{I}_{\mathrm{AM}}(0) ; \mathrm{I}_{\mathrm{MT}}(0) ; \mathrm{I}_{\mathrm{HT}}(0) ; \mathrm{I}_{\mathrm{AT}}(0) ; \mathrm{I}_{\mathrm{HMT}}(0)\right.$; $\left.\mathrm{I}_{\mathrm{AMT}}(0) ; \mathrm{IV}_{\mathrm{V}}(0)\right\} \in \Psi$. Then the solution set $\left\{\mathrm{S}_{\mathrm{H}} ; \mathrm{S}_{\mathrm{V}} ; \mathrm{I}_{\mathrm{M}} ; \mathrm{I}_{\mathrm{H}} ; \mathrm{I}_{\mathrm{A}} ; \mathrm{I}_{\mathrm{T}} ; \mathrm{I}_{\mathrm{HM}} ; \mathrm{I}_{\mathrm{AM}} ; \mathrm{I}_{\mathrm{MT}} ; \mathrm{I}_{\mathrm{HT}} ; \mathrm{I}_{\mathrm{AT}} ; \mathrm{I}_{\mathrm{HMT}} ; \mathrm{I}_{\mathrm{AMT}} ; \mathrm{IV}_{\mathrm{V}}\right\}(\mathrm{t})$ is positive for all time $\mathrm{t}$ $>0$. 
Proof. Consider the first equation of 2.2.1 at time $\mathrm{t}$

$$
\frac{d S_{H}}{d t}=\Lambda_{H}+r_{m} I_{M}+r_{t} I_{T}-\lambda_{a h} S_{H}-\lambda_{m h} S_{H}-\lambda_{t h} S_{H}-d_{n} S_{H}
$$

then

$$
\begin{gathered}
\frac{d S_{H}}{d t} \geq-\left(\lambda_{a h}+\lambda_{m h}+\lambda_{t h}+d_{n}\right) S_{H} \\
\int \frac{d S_{H}}{S_{H}} \geq-\int\left(\lambda_{a h}+\lambda_{m h}+\lambda_{t h}+d_{n}\right) d(t) \\
S_{H}(t) \geq S_{H}(0) e^{-\int\left(\lambda_{a h}+\lambda_{m A}+\lambda_{t h}+d_{n}\right) d(t)} \geq 0
\end{gathered}
$$

From the second equation of 2.2.1 at time $t$

$$
\frac{d I_{M}}{d t}=\lambda_{m h} S_{H}+r_{t} I_{T M}-r_{m} I_{M}-e_{m}^{a} \lambda_{a h} I_{M}-\lambda_{t h} I_{M}-d_{n} I_{M}-d_{m} I_{M}
$$

then

$$
\begin{gathered}
\frac{d I_{M}}{d t} \geq-\left(r_{m}+e_{m}^{a} \lambda_{a h}+\lambda_{t h}+d_{n}+d_{m}\right) I_{M} . \\
\frac{d I_{M}}{I_{M}} \geq-\int\left(r_{m}+e_{m}^{a} \lambda_{a h}+\lambda_{t h}+d_{n}+d_{m}\right) d t . \\
I_{M}(t) \geq I_{M}(0) e^{-\int\left(r_{m}+e_{m}^{a} \lambda_{a h}+\lambda_{t h}+d_{n}+d_{m}\right) d t} \geq 0 .
\end{gathered}
$$

We can proceed in a similar manner and show that all the state variables are positive for all time $t$.

Lemma 3.2. The solutions of the model 2.2.1 are uniformly bounded in a proper subset $\Psi=\Psi_{\mathrm{H}} \times \Psi_{\mathrm{V}}$

Proof. Let $\left\{\mathrm{S}_{\mathrm{H}} ; \mathrm{SV}_{\mathrm{V}} ; \mathrm{IM}_{\mathrm{M}} ; \mathrm{IH}_{\mathrm{H}} ; \mathrm{I}_{\mathrm{A}} ; \mathrm{IT}_{\mathrm{T}}\right.$; Iнм $\left.; \mathrm{I}_{\mathrm{AM}} ; \mathrm{I}_{\mathrm{MT}} ; \mathrm{IHT}_{\mathrm{H}} ; \mathrm{I}_{\mathrm{AT}} ; \mathrm{IHMT}_{\mathrm{H}} ; \mathrm{I}_{\mathrm{AMT}} ; \mathrm{IV}\right\}(\mathrm{t}) \in R_{+}^{12}$, be any solution with non-negative initial conditions. The rate of change of the total human population with time is given by:

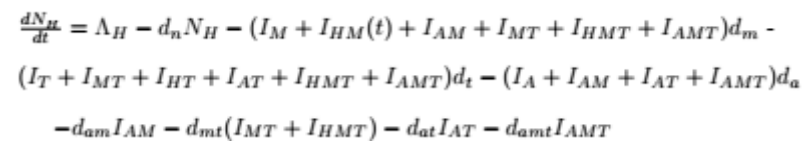

The model system 2.2.1 has a varying human population size $\frac{d N_{H}}{d t} \neq 0$ and therefore a trivial equilibrium is not feasible. Whenever, $N_{H}>\frac{\Lambda_{H}}{d_{n}}$ then $\frac{d N_{H}}{d t}<0$ Since $\frac{d N_{H}}{d t}$ is bounded by $\Lambda_{H}-d_{n} N_{H}$, a standard comparison theorem by (Birkoff and Rota, 1989) shows that $0 \leq N_{H}(t) \leq N_{H}(0) e^{-d_{n}}+\frac{\Lambda_{H}}{d_{n}}\left(1-e^{-d_{n}}\right)$, where $N_{H}(0)$ represents the value of $N_{H}(\mathrm{t})$ evaluated at the initial values of the respective variables. Thus as $t \rightarrow \infty$, we have, $0 \leq N_{H}(t) \leq \frac{\Lambda_{H}}{d_{n}}$. In particular $N_{H}(t) \leq \frac{\Lambda_{H}}{d_{n}}$, if $N_{H}(0) \leq \frac{N_{H}}{d_{n}}$. This shows that $\mathrm{N}_{\mathrm{H}}$ is bounded and all the feasible solutions of the human only component of model 2.2.1 starting in the region $\Psi_{\mathrm{H}}$ approach, enter or stay in the region, where: $\Psi_{\mathrm{H}}=\left\{\left(\mathrm{S}_{\mathrm{H}}, \mathrm{I}_{\mathrm{M}}, \mathrm{I}_{\mathrm{H}}, \mathrm{I}_{\mathrm{A}}, \mathrm{I}_{\mathrm{T}}, \mathrm{I}_{\mathrm{MH}}, \mathrm{I}_{\mathrm{MA}}, \mathrm{I}_{\mathrm{MT}}, \mathrm{I}_{\mathrm{HT}}, \mathrm{I}_{\mathrm{TA}}, \mathrm{I}_{\mathrm{MHT}}, \mathrm{I}_{\mathrm{MAT}}\right): N_{H}(t) \leq \frac{N_{H}}{d_{n}}\right\}$

Similarly let $\left\{\left(\mathrm{S}_{\mathrm{V}} ; \mathrm{IV}_{\mathrm{v}}\right) \in R_{+}^{2}\right\}$, be any solution with non-negative initial conditions. The rate of change of the total vector population with time is given by: $\frac{d N_{v}}{d t}=\Lambda_{v}-\left(S_{v}(t)-I_{v}(t)\right) d_{v} \cdot \frac{d N_{v}}{d t} \neq 0$ and therefore a trivial equilibrium is not feasible. Whenever $N_{v}>\frac{\Lambda_{v}}{d_{v}}$, then $\frac{d N_{v}}{d t}<0$. Since $\frac{d N_{v}}{d t}$ is bounded by $\Lambda_{v}-d_{v} N_{v}$, a standard comparison theorem by Birkoff and Rota (1989), shows that $0 \leq N_{v}(t) \leq N_{v}(0) e^{-d_{v}}+\frac{\Lambda_{v}}{d_{v}}\left(1-e^{-d_{v}}\right)$, where $\mathrm{Nv}_{\mathrm{v}}(0)$ represents the value of $\mathrm{N}_{\mathrm{v}}(\mathrm{t})$ evaluated at the initial values of the respective variables. Thus as $t \rightarrow \infty, 0 \leq N_{v}(t) \leq \frac{\Lambda_{v}}{d_{v}}$. In particular, $N_{v}(t) \leq \frac{\Lambda_{v}}{d_{v}}$, if $N_{v}(0) \leq \frac{N_{v}}{d_{v}}$. This shows that $N_{v}$ is bounded and all the feasible solutions of the vector only component of model 2.2.1 starting in the region $\Psi_{\mathrm{V}}$ approach, enter or stay in the region, where: $\Psi_{\mathrm{V}}$ $=\left\{\left(\mathrm{S}_{\mathrm{v}}, \mathrm{IV}_{\mathrm{v}}\right): \mathrm{N}_{\mathrm{v}} \leq \frac{\Lambda_{v}}{d_{v}}\right\}$.

\section{HIV/AIDS-only model}

Before analyzing the full model (HIV/AIDS, Malaria and TB), it is instructive to gain insights into the dynamics of the HIV/AIDS only model, HIV/AIDS-malaria co infection model and the HIV/AIDS-TB co infection model. The model with HIV/AIDS only is obtained by setting $\mathrm{I}_{\mathrm{M}}=\mathrm{S}_{\mathrm{V}}=\mathrm{I}_{\mathrm{T}}=\mathrm{I}_{\mathrm{HM}}=\mathrm{I}_{\mathrm{AM}}=\mathrm{I}_{\mathrm{MT}}=\mathrm{I}_{\mathrm{HT}}=\mathrm{I}_{\mathrm{AT}}=\mathrm{I}_{\mathrm{HMT}}=\mathrm{I}_{\mathrm{AMT}}=\mathrm{IV}_{\mathrm{V}}=0$ and obtain 


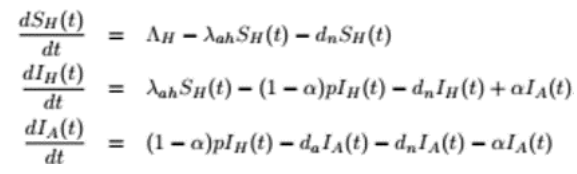

where, $N_{H}=S_{H}+I_{H}+I_{A}$, and $\lambda_{a h}=\frac{\beta_{a}(1-\delta) c_{1} I_{H}}{N_{H}}$. For this model, it can be shown that the region, $\Omega_{H}=\left\{\left(S_{H}, I_{H}, I_{A}\right) \in \mathbb{R}_{+}^{3}: N_{H} \leq \frac{\Lambda_{\mu}}{d}\right\}$, is positively-invariant and attracting. Thus, 4.0.1 is mathematically well posed and its dynamics can be considered in $\Omega_{H}$.

\subsection{The basic reproduction number $\left(R_{0}\right)$}

The basic reproduction number $\mathrm{R}_{0}$ is defined as the average number of secondary infections an infectious individual would cause over his infectious period in an entirely susceptible population. The basic reproduction number $\mathrm{R}_{\mathrm{H}}$ for the HIV/AIDS only model is defined as the number of secondary HIV/AIDS infections due to a single HIV/AIDS infective individual. When $\mathrm{R}_{\mathrm{H}}<1$, then an infectious individual is causing, on average, less than one new infection and thus the disease does not invade the population. On the other hand, when $R_{H}>1$ then an infectious individual is causing, on average, more than one new infection and thus the disease invades and persist in the population.

Table 1: Parameter Values for the HIV/AIDS - Malaria Co Infection Model

\begin{tabular}{|l|l|l|l|}
\hline Symbol & Parameter & Value $\left(\mathrm{yr}^{-1}\right)$ & Source \\
\hline \hline$\Lambda_{H}$ & Recruitment rate of humans & $0.4 \times 40 \times 10^{6}$ & $\begin{array}{l}\text { Kenya demographics } \\
\text { profile [12] }\end{array}$ \\
\hline$d_{n}$ & Natural death rate of humans & 0.016667 & $\begin{array}{l}\text { Kenya demographics } \\
\text { profile [12] }\end{array}$ \\
\hline$d_{a}$ & HIV/AIDS-induced death rate & 0.4 & WHO report [22] \\
\hline$p$ & $\begin{array}{l}\text { Progresion rate from HIV } \\
\text { to AIDS (untreated) }\end{array}$ & 0.1 & $\begin{array}{l}\text { Baryama, F. and } \\
\text { Mugisha, T.[5] }\end{array}$ \\
\hline$\alpha$ & $\begin{array}{l}\text { Proportion of the HIV/AIDS } \\
\text { victims treated }\end{array}$ & 0.6 & $\begin{array}{l}\text { Kenya NACC } \\
\text { report [13] }\end{array}$ \\
\hline$\beta_{a}$ & $\begin{array}{l}\text { Transmission probability } \\
\text { of HIV/AIDS }\end{array}$ & 0.019 & $\begin{array}{l}\text { Baryama, F. and } \\
\text { Mugisha, T. [5] }\end{array}$ \\
\hline$c_{1}$ & $\begin{array}{l}\text { Per capita number of sexual } \\
\text { contacts }\end{array}$ & 9 & $\begin{array}{l}\text { Kenya NACC } \\
\text { report [13] }\end{array}$ \\
\hline$\delta$ & Effectiveness of counseling & Variable & \\
\hline
\end{tabular}

\subsubsection{Local stability of disease-free equilibrium (DFE)}

The model 4.0.1 has a DFE, obtained by setting the right-hand sides of the equations in the model to zero given by $E_{0}=S_{H}^{*}+I_{H}^{*}+I_{A}^{*}$ $=\left(\frac{\Lambda_{H}}{d_{n}}, 0,0\right)$ Define $\mathrm{F}_{\mathrm{i}}$ as the rate of appearance of new infections in the class or compartment $\mathrm{i}$ and $v_{i}=v_{i}^{-}-v_{i}^{+}$, where $v_{i}^{-}$is the rate of transfer of individuals out of compartment $\mathrm{i}$, and $v_{i}^{+}$is the rate of transfer of individuals into compartment $\mathrm{i}$ by all other means. Therefore:

$$
\begin{aligned}
& \mathcal{F}_{i}=\left(\begin{array}{c}
\frac{\beta_{a}\left(1-\delta c_{i} I_{n}\right.}{N_{n_{0}}} S_{H} \\
0
\end{array}\right) \\
& \text { and } \\
& \mathcal{V}_{i}=\left(\begin{array}{c}
(1-\alpha) p I_{H}+d_{n} I_{H}-\alpha I_{A} \\
d_{a} I_{A}+d_{n} I_{A}+\alpha I_{A}-(1-\alpha) p I_{H}
\end{array}\right) \\
& \text { The Jacobian of } \mathcal{F}_{i} \text { and } \mathcal{V}_{i} \text { at the disease-free equilibrium denoted by } F \text { and } V \text { respectively } \\
& \text { is given by: } \\
& F=\left(\begin{array}{cc}
\beta_{a}(1-\delta) c_{1} & 0 \\
0 & 0
\end{array}\right) \\
& \text { and } \left.\begin{array}{cc}
(1-\alpha) p+d_{n} & -\alpha \\
-(1-\alpha) p & d_{a}+d_{n}+\alpha
\end{array}\right) \\
& V=\left(\begin{array}{cc} 
\\
V
\end{array}\right.
\end{aligned}
$$

The basic reproduction number $R_{H}$ is by definition the spectral radius of the matrix $F V^{-1}$ and is given by:

$$
R_{H}=\frac{c_{1}(1-\delta)\left(\alpha+d_{a}+d_{n}\right) \beta_{a}}{\left(\alpha d_{n}+d_{a} d_{n}+d_{n}^{2}+d_{a} p-\alpha d_{a} p+d_{n} p-\alpha d_{n} p\right)}
$$

It measures the average number of new HIV infections generated by a single HIV/AIDS infected individual in a population where a certain fraction of infected individuals are treated and counseled.

Lemma 4.1. The DFE of the HIV-only model is locally asymptotically stable (LAS) if $\mathrm{R}_{\mathrm{H}}<1$, and unstable otherwise. Lemma 4.1 follows from Theorem two by Van, P. and Watmough, J. (2002). This lemma is illustrated graphically in figure 1 showing total infectives $\left(\mathrm{I}_{\mathrm{H}}+\mathrm{I}_{\mathrm{A}}\right)$ against time in years 


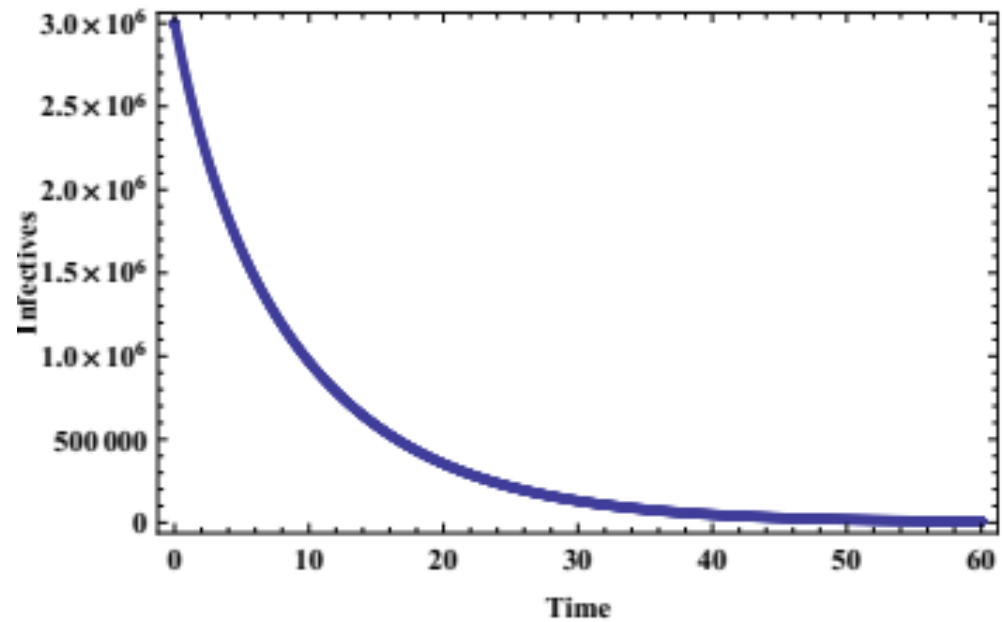

Fig. 1: Reproduction Number $\left(\mathrm{R}_{\mathrm{H}}\right)=0.517234$.

\section{Simulating the role of counseling and treatment}

From equation 4.1.1, it is evident that strategies for the reduction of HIV/AIDS infections in humans should target the reduction of the parameter $c_{1}$ (per capita number of sexual contacts) through counseling. Effective counseling where $\delta=1$, reduces the value of $c_{1}$ and $R_{H}$ to zero eliminating the HIV/AIDS problem. The graph of $\mathrm{R}_{\mathrm{H}}$ against $\mathrm{c}_{1}$ is shown in figure $2 \mathrm{a}$.

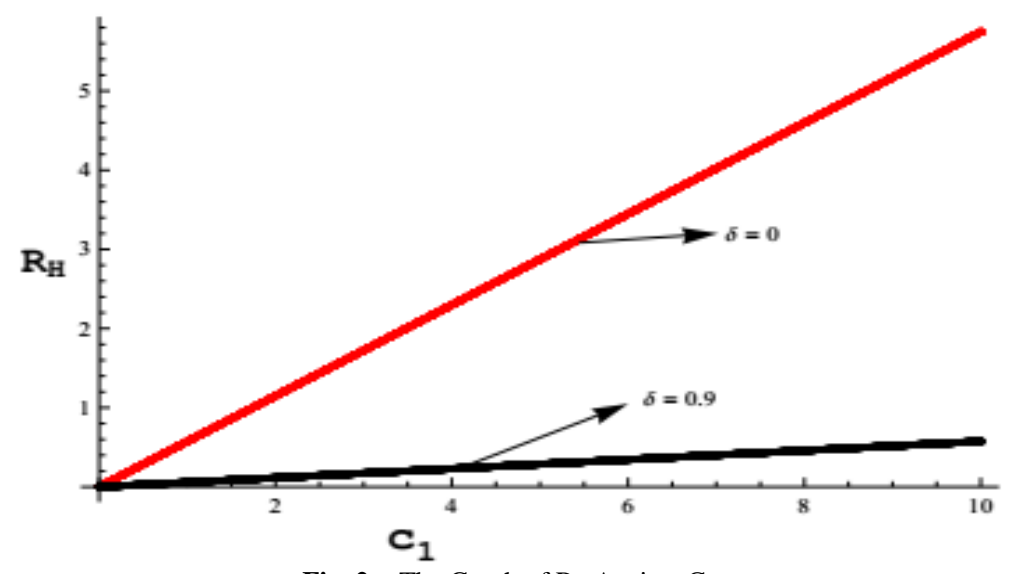

Fig. 2a: The Graph of $\mathrm{R}_{\mathrm{H}}$ Against $\mathrm{C}_{1}$

This figure shows that effective counseling $(\delta=0.9)$ reduces the value of $\mathrm{R}_{\mathrm{H}}$ to less than unity and therefore very effective in controlling the HIV/AIDS problem. This is further illustrated in terms of the infective population sizes against time in years as shown in figure $2 \mathrm{~b}$ where $\delta=0$ and $\mathrm{R}_{H}=5.17234$ and figure $2 \mathrm{c}$ where $\delta=0.9$ and $\mathrm{R}_{\mathrm{H}}=0.517234$.

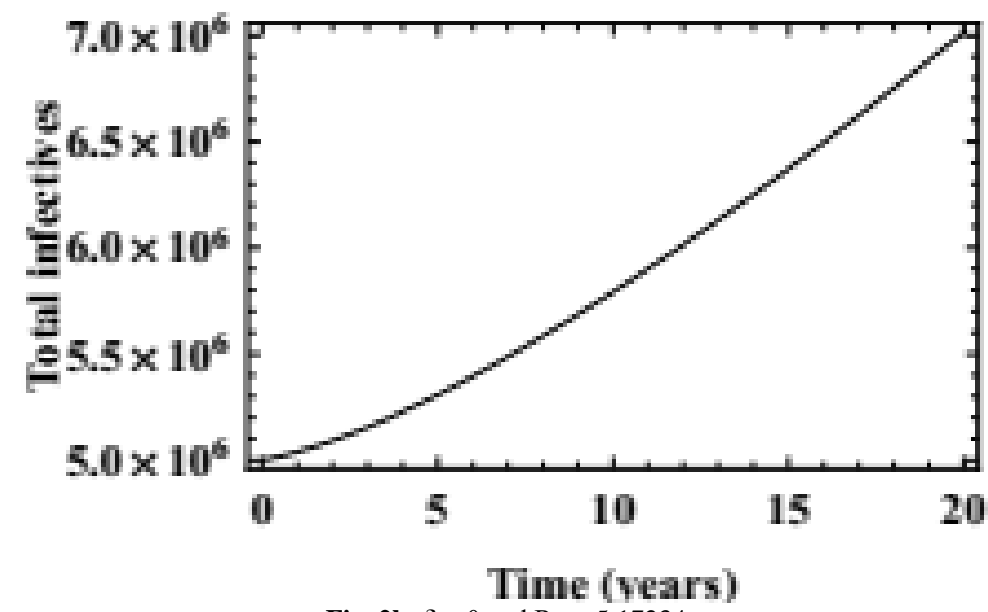

Fig. 2b: $\delta=0$ and $\mathrm{R}_{\mathrm{H}}=5: 17234$. 


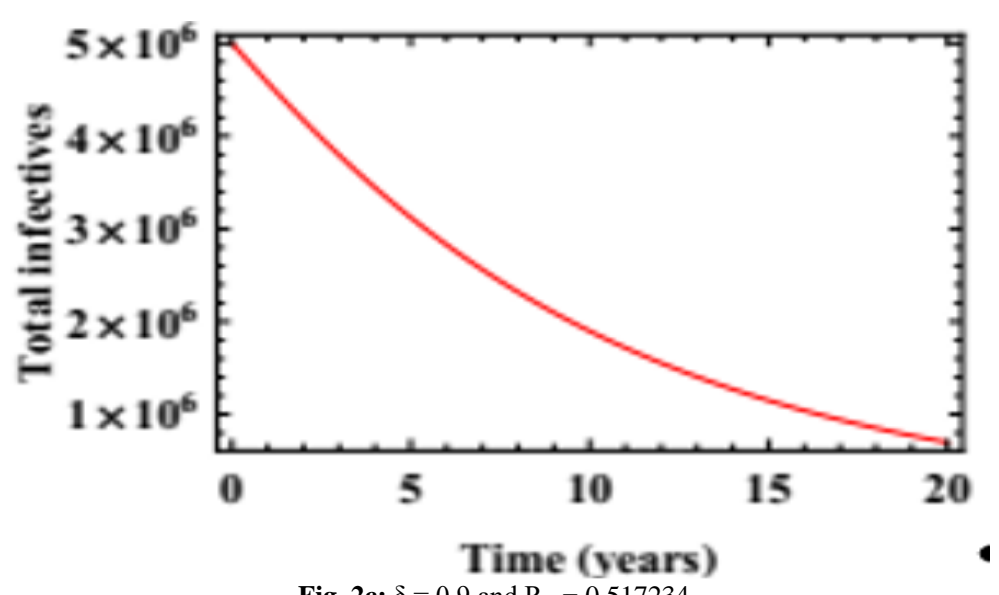

Fig. 2c: $\delta=0.9$ and $\mathrm{R}_{\mathrm{H}}=0.517234$.

Figure 3a combines the parameters for ARV treatment $(\alpha)$ and counseling $(\delta)$ within the same graph. The graph shows that ARV treatment without effective counseling $(\alpha=0.6$ and $\delta=0)$, increases the value of $\mathrm{R}_{\mathrm{H}}$, worsening the HIV/AIDS problem, however ARV treatment coupled with effective counseling $(\alpha=0.6$ and $\delta=0.9)$ reduces the value of $\mathrm{R}_{\mathrm{H}}$ to a level below one eliminating the disease. This figure suggests that there is a threshold level of counseling below which ARV treatment is disastrous. Above the threshold level, ARV treatment and counseling would be very effective

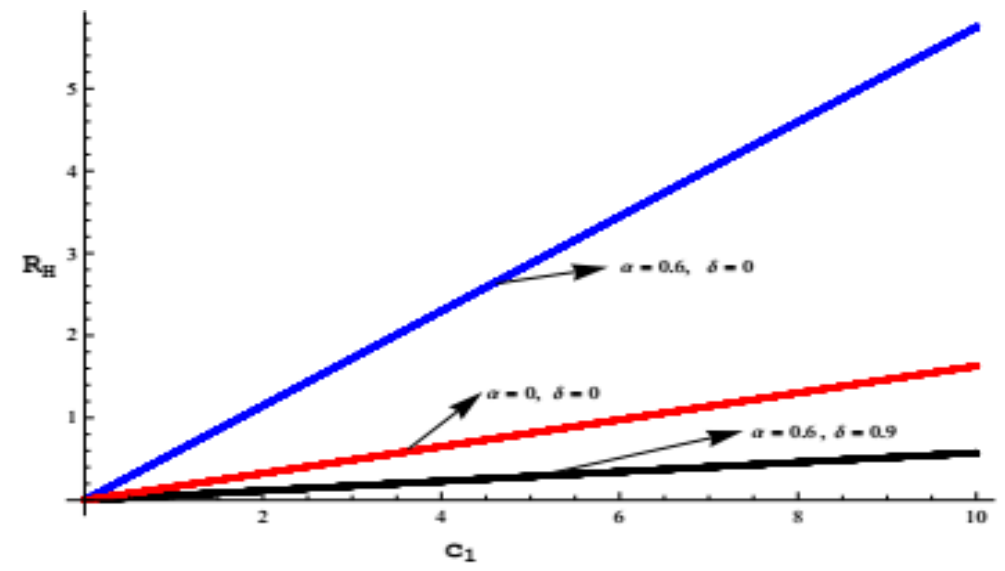

Fig. 3a: Graph of RH Against Per Capita Number of Sexual Contacts.

When the proportion of those receiving ARV treatment without effective counseling increases, the value of $\mathrm{R}_{H}$ also increases to a level above one, however effective counseling maintains the value of $\mathrm{R}_{H}$ below unity as shown in figure $3 \mathrm{~b}$. Therefore strategies for the control of HIV/AIDS should emphasize counseling and not only treatment.

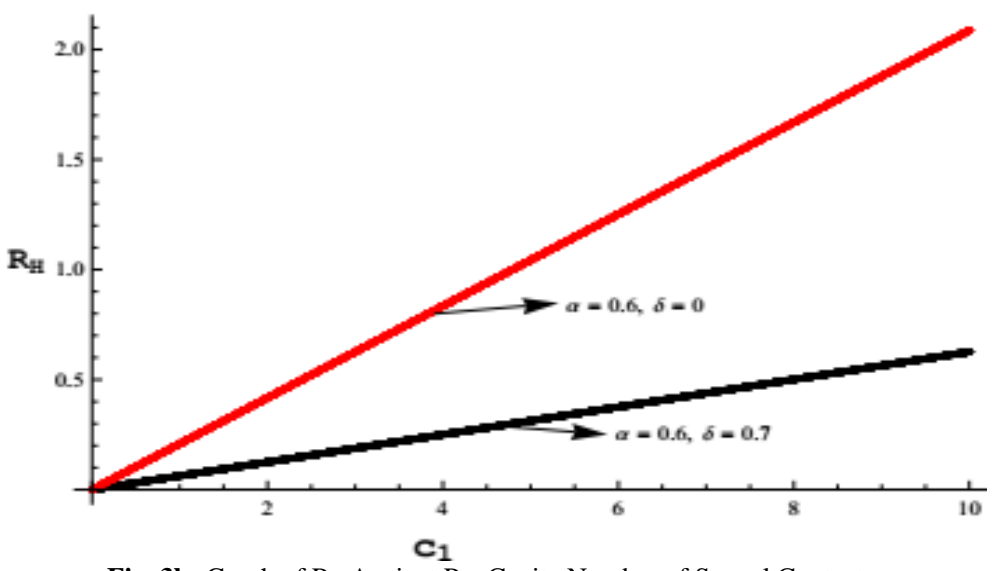

Fig. 3b: Graph of $R_{H}$ Against Per Capita Number of Sexual Contacts.

To further investigate the potential impact of counseling and treatment on disease progression, we carry out sensitivity analysis of the reproduction number with respect to counseling and treatment. The sensitivity index of $\mathrm{R}_{H}$ with respect to $\delta$ is given by

$R_{H}^{\delta}=-\frac{\delta}{1-\delta}$

The negative sign in equation 5.0.1 indicates that there is an expected decline in the rate of new HIV/AIDS infections when counseling is scaled up. Similarly, the sensitivity index of $\mathrm{R}_{\mathrm{H}}$ with respect to $\alpha$ is given by 


$$
R_{H}^{\alpha}=\frac{\alpha A_{1}\left\{-\frac{\beta_{a} c_{1} A_{2} A_{3}(1-\delta)}{A_{1}^{2}}+\frac{\beta_{a} c_{1}(1-\delta)}{A_{1}}\right\}}{\beta_{a} c_{1} A_{2}(1-\delta)}
$$

$A_{1}=\alpha d_{n}+d_{a} d_{n}+d_{n}^{2}+d_{a} p-\alpha d_{a} p+d_{n} p-\alpha d_{n} p$

$A_{2}=\alpha+d_{a}+d_{n}$

$A_{3}=d_{n}-d_{a} p-d_{n} p$

Numerical simulations shows that the sensitivity index of $\mathrm{R}_{\mathrm{H}}$ with respect to treatment is positive indicating that an increase in the proportions of those treated leads to an increase in new HIV cases as shown in figure 4.

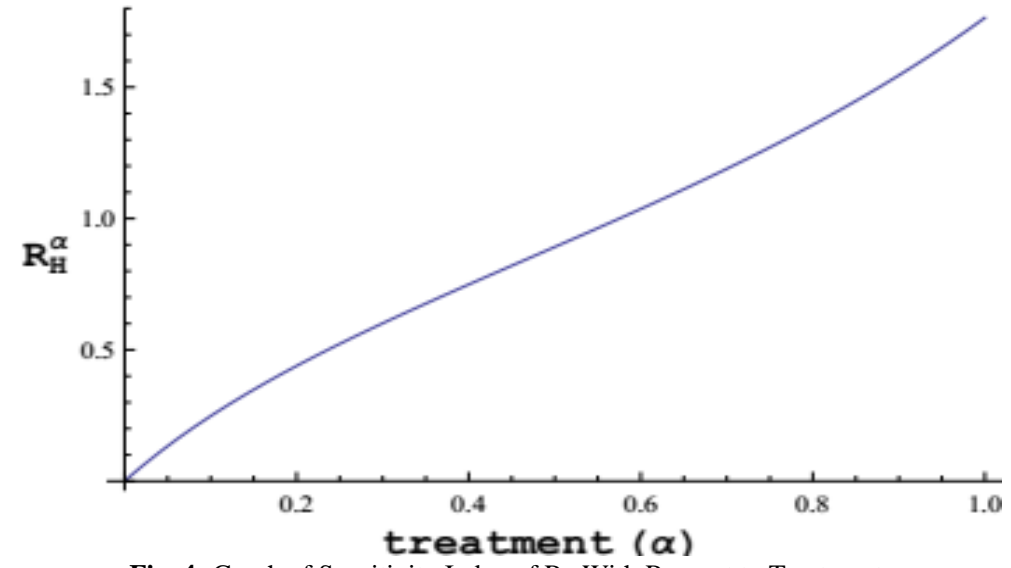

Fig. 4: Graph of Sensitivity Index of $R_{H}$ With Respect to Treatment.

Biologically, lemma 4.1 implies that HIV/AIDS can be eliminated from the community when $R_{H}<1$. This is only true if the initial sizes of the sub-populations of the model are in the basin of attraction of $\mathrm{E}_{0}$. To ensure that elimination of the virus is independent of the initial sizes of the sub-populations, it is necessary to show that the DFE is globally asymptotically stable.

\subsubsection{Global stability of disease-free equilibrium (DFE)}

The global asymptotic stability (GAS) of the disease-free state of the model is investigated using the theorem by Castillo-Chavez et al. (2002). The model is rewritten as follows:

$$
\begin{aligned}
& \frac{d X}{d t}=H(X, Z) \\
& \frac{d Z}{d t}=G(X, Z), \quad G(X, 0)=0
\end{aligned}
$$

where the components of the column-vector $X \in \mathrm{R}^{\mathrm{m}}$ denote the uninfected population and the components of $\mathrm{Z} \in \mathrm{R}^{\mathrm{n}}$ denote the infected population. $E_{0}=\left(X^{*}, 0\right)$ denotes the disease free equilibrium of this system. The fixed point $E_{0}=\left(X^{*}, 0\right)$ is globally asymptotically stable (GAS) equilibrium for this system provided that $\mathrm{R}_{0}<1$ and the following two conditions satisfied:

(H1) For $\frac{d X}{d t}=H(X, 0), X^{*}$ is globally asymptotically stable

(H2) $G(X, Z)=P Z-\widehat{G}(X, Z), \hat{G}(X, Z) \geq 0$ for $(X, Z) \in \Omega_{H}$,

where $P=D_{Z} G\left(X^{*}, 0\right)$ is an M-matrix (the off diagonal elements of $\mathrm{P}$ are non negative)

and $\Omega_{H}$ is the region where the model makes biological sense. The disease-free equilibrium

is now denoted as $E^{0}=\left(X^{*}, 0\right), X^{*}=\frac{\Lambda_{n}}{d_{n}}$

Theorem 5.1. The fixed point $E_{0}=\left(X^{*}, 0\right)$ is a globally asymptotically stable equilibrium of system 4.0 .1 provided that $\mathrm{R}_{\mathrm{H}}<1$ and the assumptions $\mathrm{H} 1$ and $\mathrm{H} 2$ are satisfied.

Proof. From the system 4.0.1

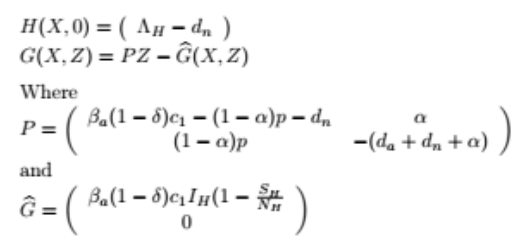

Notice that $\hat{G}=(X, Z) \geq 0$ in $\Omega_{\mathrm{H}}$. Therefore the DFE of model 4.0.1 is globally asymptotically stable if $\mathrm{R}_{\mathrm{H}}<1$. This shows that HIV/AIDS will be completely eliminated from the community if the epidemiological threshold, $R_{H}$ can be brought to a value less than unity independent of the initial sizes of the sub-populations as shown numerically in figure 5 . 


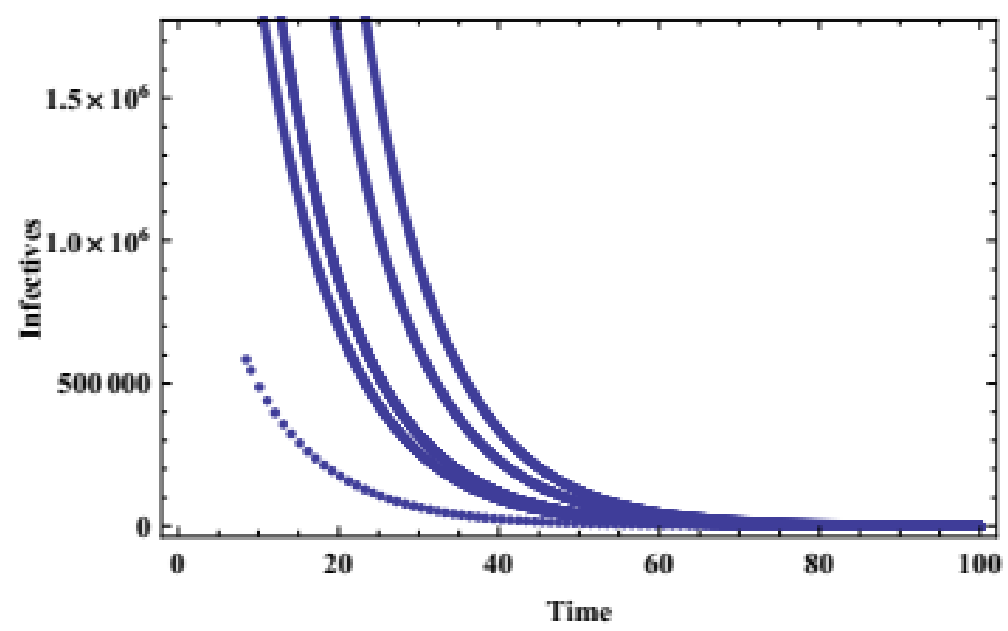

Fig. 5: Global Stability of Disease-Free Equilibrium (DFE).

\section{Existence and stability of the endemic equilibrium}

To find conditions for the existence of an equilibrium for which HIV/AIDS is endemic in the population, the steady states of the system 4.0.1 are determined by solving $E_{1}=f\left(S_{H}^{*}+I_{H}^{*}+I_{A}^{*}\right)=0$. The equations for the population proportions are considered by first scaling the sub-populations for $\mathrm{S}_{\mathrm{H}}$; $\mathrm{I}_{\mathrm{H}}$ and $\mathrm{I}_{\mathrm{A}}$ using the following set of new variables: $s_{H}=\frac{S_{H}}{N_{H}}, i_{H}=\frac{I_{H}}{N_{H}}, i_{A}=\frac{I_{A}}{N_{H}}$.

The system 4.0.1, is therefore given by

$$
\begin{aligned}
\frac{d s_{H}}{d t} & =\frac{\Lambda_{H}}{N_{H}}-\lambda_{a h} s-s\left(\frac{\Lambda_{H}}{N_{H}}-d_{a} i_{A}\right) \\
\frac{d i_{H}}{d t} & =\lambda_{a h} s-(1-\alpha) p i_{H}+\alpha i_{A}-i_{H}\left(\frac{\Lambda_{H}}{N_{H}}-d_{a} i_{A}\right) \\
\frac{d i_{A}}{d t} & =(1-\alpha) p i_{H}-d_{a} i_{A}-\alpha i_{A}-i_{A}\left(\frac{\Lambda_{H}}{N_{H}}-d_{a} i_{A}\right) \\
i_{A}^{*} & =\frac{(1-\alpha) p i_{H}}{d_{a}+d_{n}+\alpha}
\end{aligned}
$$

At the steady states, $\frac{d N_{H}}{d t}=0$ and $\frac{\Lambda_{H}}{N_{H}}-d_{a} i_{A}=d_{n}$. By setting $s_{H}=\left(1-i_{H}-i_{A}\right)$, the coordinates of the endemic equilibrium of the system 6.0 .1 satisfy:

$$
i_{H}=\frac{\left\{\beta_{1}(1-\delta) c_{1} \pi_{1}+\alpha(1-\alpha) p\right\}-\left\{d_{n} \pi_{1}+p \pi_{1}(1-\alpha)\right\}}{\beta_{1}(1-\delta) c_{1}\left\{\pi_{1}+p(1-\alpha)\right\}}
$$

where $\pi=\alpha+d_{a}+d_{n}$. Since $I_{H}^{*}$ is positive, then $\left\{\beta_{1}(1-\delta) c_{1} \pi_{1}+\alpha(1-\alpha) p\right\}>\left\{d_{n} \pi_{1}+p \pi_{1}(1-\alpha)\right\}$ which indicates the existence of only one unique endemic equilibrium point suggesting that there is no bifurcation. When $\left\{\beta_{1}(1-\delta) c_{1} \pi_{1}+\alpha(1-\alpha) p\right\}<\left\{d_{n} \pi_{1}+p \pi_{1}(1-\alpha)\right\}$, then the model has no positive equilibrium. It can also be verified by a theorem by Castillo Chavez and Song (2002), that the model 6.0 .1 has a unique endemic equilibrium which is LAS whenever $\mathrm{R}_{\mathrm{H}}>1$ as shown below. The equations in 6.0.1 are solved in terms of the force of infection at steady-state $\lambda_{\text {ah, }}$ given by

$\lambda_{a h}^{*}=\frac{\beta_{a} c_{1}(1-\delta) I_{H}^{*}}{S_{H}^{*}+I_{H}^{*}+I_{A}^{*}}$

Setting the right hand sides of the model 4.0.1 to zero and noting that $\lambda_{a h}=\lambda_{a h}^{*}$ at equilibrium gives

$$
\begin{aligned}
S_{H}^{*} & =\frac{\Lambda_{H}}{\lambda_{a h}+d_{n}} \\
I_{H}^{*} & =\frac{\lambda_{a h} S_{H}^{*}+\alpha I_{A}^{*}}{d_{n}+(1-\alpha) p} \\
I_{A}^{*} & =\frac{(1-\alpha) p I_{H}^{*}}{d_{a}+d_{n}+\alpha}
\end{aligned}
$$

Using 6.0.5 in the expression for $\lambda_{a h}^{*}$ in 6.0.4 shows that the nonzero endemic equilibria of the model satisfy

$a_{11} \lambda_{a h}^{*}-a_{12}=0$ 
where $\mathrm{a}_{12}=\mathrm{R}_{\mathrm{H}}-1$ and $a_{11}=\frac{a_{11}}{\lambda_{a h}}$. It is clear that $\mathrm{a}_{11}>0$, and $\mathrm{a}_{12}>0$ for $\mathrm{R}_{\mathrm{H}}>1$. Thus, the linear system 4.0.1 has only one unique positive solution, given by $a_{a h}^{*}=\frac{a_{12}}{a_{11}}$, whenever $\mathrm{R}_{\mathrm{H}}>1$. This result is numerically illustrated in figure $6 \mathrm{a}$ where $\mathrm{R}_{\mathrm{H}}=1.03447$ and $6 \mathrm{~b}$ where $\mathrm{R}_{\mathrm{H}}=5.17234$

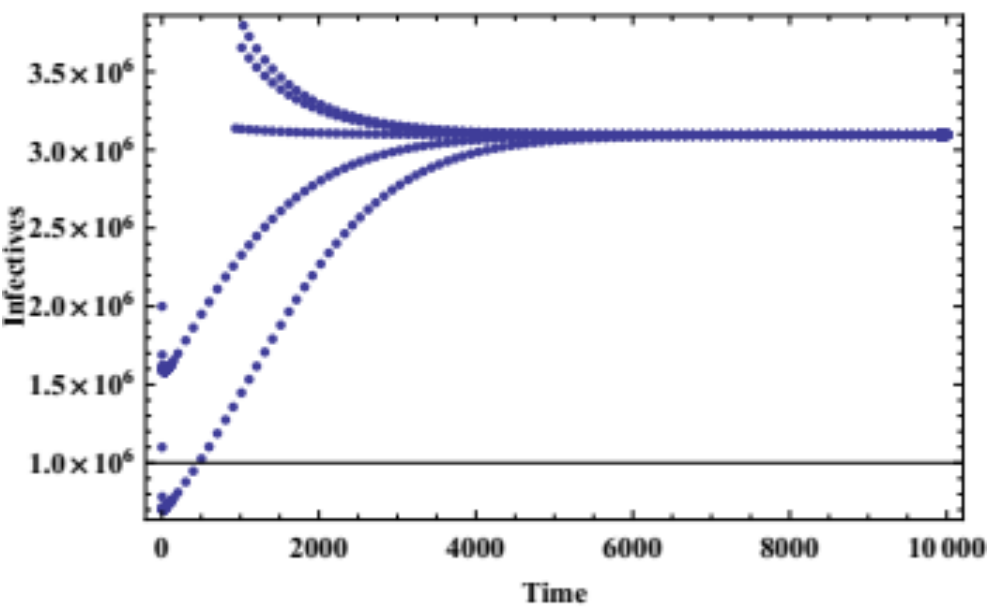

Fig. 6a: Global Stability of the Endemic Equilibrium (EE).

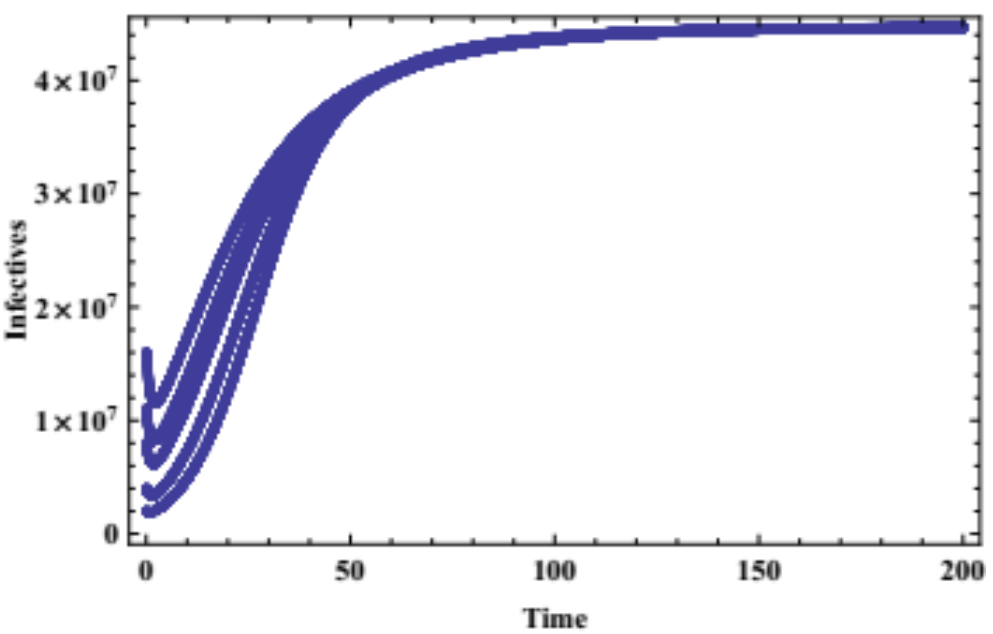

Fig. 6b: Global Stability of the Endemic Equilibrium (EE).

These results are summarized in lemma 6.1.

Lemma 6.1. The HIV-only model 4.0.1 has a unique endemic equilibrium whenever $\mathrm{R}_{\mathrm{H}}>1$, and no endemic equilibrium otherwise. When an endemic equilibrium point of a disease is unique and stable, then we would expect easier management of the disease because there is no bifurcation and therefore reducing the value of $R_{H}$ to less than one eliminates the disease.

\section{Conclusion}

The HIV-only model 4.0.1 has a globally-asymptotically stable DFE whenever $R_{H}>1$, and a unique endemic equilibrium point whenever $R_{H}>1$. This study shows that effective counseling reduces the value of the reproduction number for HIV/AIDS (RH) to less than unity eliminating the HIV/AIDS problem. When the proportion of those receiving ARV treatment without effective counseling increases, the value of $R_{H}$ also increases to a level above one, however effective counseling maintains the value of $R_{H}$ below unity therefore strategies for the control of HIV/AIDS should emphasize counseling and not only treatment.

\section{Acknowledgements}

The authors are very grateful to Dr. Ochieng Ombaka (Chuka University, Department of Physical sciences) for many excellent comments that have enhancedthe model as well as the clarity of the paper.

\section{References}

[1] Abu-Raddad, L, Patnaik P, and J. Kublin, (2006), "Dual infection with HIV and malaria fuels the spread of both diseases in Sub-Saharan Africa", Science, 314(5805), 1603- 1606. https://doi.org/10.1126/science.1132338.

[2] Allman E, and Rhodes J, (2004) "An introduction to Mathematical models in Biology", Cambridge University press: New York.

[3] Anderson and May R, (1993). "Infectious Diseases of Humans: Dynamics and Control", Oxford University Press: United Kingdom. 
[4] Audu E, Onwujekwe D, Onubogu C, Adedoyin J, Onyejepu N, Mafe A, Onyewuche J, Oparaugo C, Enwuru C, Aniedobe M, Musa A, and Idigbe $\mathrm{E}$, (2005), "Impact of co infections of tuberculosis and malaria on the CD4+ cell counts of HIV patients in Nigeria", Annals of African Medicine, 4(1): 10-13.

[5] Baryama F, and Mugisha T, (2007), "Comparison of single - stage and staged progression models for HIV/AIDS models", International Journal of Mathematics and Mathematical sciences, 12(4):399 - 417. https://doi.org/10.1155/2007/18908.

[6] Bhunu C, Garira W, and Mukandavire Z, (2009), "Modeling HIV/AIDS and Tuberculosis Co infection", Bulletin of Mathematical Biology, 71: 17451780. https://doi.org/10.1007/s11538-009-9423-9.

[7] Center for Disease Control and Prevention (CDC), (2013), "Incorporating HIV prevention into the medical care of persons living with malaria": MMWR 2006;55(No. RR-14):1-17. http: www.cdc.gov/malaria/facts.htm, Accessed August 22nd, 2013.

[8] Diekmann O, and Heesterbeek J, (2000). "Mathematical epidemiology of infectious diseases". Chichester: Wiley,

[9] Granich R, Gilks C, Dye C, Decock K and Wlliam B, (2008), "Universal Voluntary HIV testing with immediate antiretroviral therapy as a strategy for elimination of HIV transmission", Journal of Mathematical Biology, 28(1):365382.

[10] Hohman S, and Kim K, (2009), "The impact of HIV and malaria co infection: What is known and suggested avenues for further study". Interdisciplinary perspectives on infectious diseases, 201(8): 617-654. https://doi.org/10.1155/2009/617954.

[11] Kamal B, David M, Svetlana R, Ana M, Tameru F, and Sharquetta T, (2007), "Mathematical Model of HIV and Malaria Co Infection in SubSaharan Africa" Alabama State University: USA, AL (5), 361-371.

[12] Kenya Demographics profile, (2014), Accesed on 3rd August 2015 at www: indexmundi.com /Kenya/demographicsprofile2014,

[13] Kenya National AIDS Control Council Report, (2014), http://www.kaisernetwork.org, Accessed on 03/08/2015.

[14] Kirschner D, (1999), "Dynamics of co infection with Mycobacterium tuberculosis and HIV-1", Theory of Population Biology, 55: 94109. https://doi.org/10.1006/tpbi.1998.1382.

[15] Kivihya L, Ochola L, Otieno G, and Muthami L, "Clinical and immunological markers in Kenyan pulmonary tuberculosis patients with and without HIV- 1". East African Medical Journal, (1994), 71(24): 373-375.

[16] Kramer A, Mirjam K, and Klaus K, (2010), "Modern infectious disease epidemiology". In: Springer (Ed.). Statistics for biology and health, Science and Business Media, Germany LLC, 210 - 219. https://doi.org/10.1007/978-0-387-93835-6.

[17] Lih-Ing W, Zhilan F, and Carlos C, "Modeling TB and HIV co infections", Mathematical Biosciences and Engineering, (2009), 6(4), 815837. https://doi.org/10.3934/mbe.2009.6.815.

[18] Martin D, Sim J, and Sole G, CD4+, lymphocyte count in African patients co infected with HIV and tuberculosis, Journal of Acquired Immune Deficiency Syndrome, (1995), 8:386-391. International Journal of Applied Mathematical Research https://doi.org/10.1097/00042560-199504000$\underline{00010 .}$

[19] Mukandavire Z, Gumel A, Garira W, and Tchuenche J, (2009), "Mathematical analysis of a model for HIV Malaria co infection", Mathematical biosciences and engineering, 6(2): 333-362. https://doi.org/10.3934/mbe.2009.6.333.

[20] Oluwaseun S, Chandra N, and Abba B, (2008), "Mathematical analysis of the transmission dynamics of HIV/TB co infection in the presence of treatment", Mathematical biosciences and Engineering, 1: 145-174. https://doi.org/10.3934/mbe.2008.5.145.

[21] Ronald R, (1911). "The Prevention of Malaria", John Murray, London,

[22] World Health Organization (WHO), (2008), "Malaria and HIV interactions and their implications for Public Health Policy", WHO Press, Geneva, Switzerland.

[23] World Health Organization (WHO), (2013), "HIV - Associated TB facts: Challenges and Key Issues", http://www.who.int/tb/challenges/hiv/, Retreived on 13th August 2013.

[24] World Health Organization (WHO), (2014): "HIV/AIDS Global Maps: Global Prevalence of HIV/AIDS, Malaria and Tuberculosis", (2013). Available online at: http://www.google.com/imgres? Accessed on 5th August 2014.

[25] Xiao D and Bossert W, (2010) "An intra-host mathematical model on interaction between HIV and malaria", Bulletin of Mathematical Biology, 72(7): 1892-1911. https://doi.org/10.1007/s11538-010-9515-6. 